\title{
X-derived marker chromosome in patient with mosaic Turner syndrome and Dandy-Walker syndrome: a case report
}

\author{
Alena S. Telepova ${ }^{1,2}$, Svetlana A. Romanenko ${ }^{1,2}$, Natalya A. Lemskaya' ${ }^{1}$, Yulia V. Maksimova ${ }^{3,4}$, Asia R. Shorina ${ }^{4}$ \\ and Dmitry V. Yudkin ${ }^{1,2^{*}}$
}

\begin{abstract}
Background: Small supernumerary marker chromosomes can be derived from autosomes and sex chromosomes and can accompany chromosome pathologies, such as Turner syndrome.

Case presentation: Here, we present a case report of a patient with mosaic Turner syndrome and Dandy-Walker syndrome carrying a marker chromosome. We showed the presence of the marker chromosome in 33.8\% of blood cells. FISH of the probe derived from the marker chromosome by microdissection revealed that it originated from the centromeric region of chromosome X. Additionally, we showed no telomeric sequences and no XIST sequence in the marker chromosome. This is the first report of these two syndromes accompanied by the presence of a marker chromosome.
\end{abstract}

Conclusion: Marker chromosome was X-derived and originated from centromeric region. Patient has mild symptoms but there is no XIST gene in marker chromosome.

Trial registration: CPG137. Registered 03 March 2017.

Keywords: Marker chromosome, Turner syndrome, Dandy-Walker syndrome, X-chromosome

\section{Background}

Small supernumerary marker chromosomes (sSMC) are structurally abnormal chromosomes. The size of sSMC is smaller than chromosome 20 on metaphase spread [1]. sSMC can be derived from autosomes and sex chromosomes. Occasionally, sSMC can accompany chromosome pathologies, such as Turner syndrome [2]. Turner syndrome is a genetic disorder caused by karyotype $45, \mathrm{X}$ with or without mosaicism [3]. Described by Henry Turner in 1938, it consists of a constellation of phenotypic findings: short stature, sexual infantilism, webbed neck, and cubitus valgus. Usually, sSMC in Turner syndrome $\left(\mathrm{sSMC}^{\mathrm{T}}\right)$ are derived from sex chromosomes [4]. Most $\mathrm{sSMC}^{\mathrm{T}}(\mathrm{X})$ form ring-chromosomes, but rare $\operatorname{sSMC}^{\mathrm{T}}(\mathrm{Y})$ are inverted duplicated/isodicentric ones. The

\footnotetext{
* Correspondence: dim@mcb.nsc.ru

${ }^{1}$ Institute of Molecular and Cellular Biology SB RAS, Lavrentieva ave. 8/2, Novosibirsk 630090, Russia

${ }^{2}$ Novosibirsk State University, Novosibirsk 630090, Russia

Full list of author information is available at the end of the article
}

frequency of $\mathrm{sSMC}^{\mathrm{T}}$ is $1: 100,000$ in a population [3]. The phenotypic variability of these mosaics is largely dependent on the size of the marker chromosome and the presence of a functioning XIST gene in the Xinactivation center (Xq13). If the marker chromosome undergoes inactivation, patients with $\mathrm{sSMC}^{\mathrm{T}}$ have a mild Turner variant phenotype [5].

In this case report we described an exceptional patient with both Turner and Dandy-Walker syndromes carrying a $\mathrm{SSMC}^{\mathrm{T}}$ derived from the centromere region of the $\mathrm{X}$ chromosome. The combination of a Dandy-Walker syndrome and $\mathrm{sSMC}^{\mathrm{T}}$ was described for the first time.

\section{Materials and methods Clinical study}

The clinical study of the patient included consultations with medical specialists such as clinical psychologists, neurologists, speech therapists and MRI research. 


\section{Metaphase preparation and GTG banding}

Metaphase chromosome preparations were obtained from lymphocyte cultures according to standard procedures [6]. GTG banding was carried out as described previously [7]. Metaphase spreads were analyzed using an Olympus BX 53 microscope and the "VideoTest Karyo 3.1" (Zenit, Russia) software.

\section{Microdissection and FISH}

Marker chromosomes were dissected as described earlier [8] using an Olympus IX 51 microscope and the micromanipulator of Eppendorf Transferman NK2. DNA of microdissected chromosomes was amplified by GenomePlex Complete Whole Genome Amplification (WGA) Kit (Sigma-Aldrich, USA). Probes were labeled with digoxigenin. Every library was obtained from one copy of marker chromosome.

FISH of microdissected probes, sorted human chromosome probes and telomeric probes was carried out as described [8]. Slides were analyzed using an Olympus BX 53 fluorescence microscope and the "VideoTest FISH 2.0" (Zenit, Russia) software.

\section{PCR screening}

PCR screening of painting probes was carried out with primers: XISTF 5'AGTGTACCTACCGCTTTGGC3' and XISTR 5'TCCTCTGCCTGACCTGCTAT3' (ref. seq. NG_016172.1) for XIST, CHXF 5'TTCTCTGTCCTGCG ACCTTG3' and CHXR 5'GCTCAAAAGACTGGGCA CCT3' (ref. seq. NC_000023.11) for centromeric region of chromosome $\mathrm{X}$ and $\mathrm{CH} 3 \mathrm{~F}$ 5'GTGACTTCCCAACCTGGA TTCT3' and CH3R 5'GATCATCCCAAAGGACATCA ACT3' (ref. seq. NG_047144.1) for CNTN6 gene on chromosome 3. Painting probes of microdissection-derived marker chromosomes and sorted chromosomes $\mathrm{X}$ and 3 were used as a template.

\section{Case presentation}

\section{Clinical study}

The patient is a 13-year-old female with growth retardation (nanism, $137 \mathrm{~cm}$ ), a short neck, low hair growth on the neck, widely spaced nipples, a shield chest, secondary sexual characteristics deficiency, and the presence of episodic hyperglycemia. Her body mass index is 25 . The parents of the patient have normal growth (mother $-175 \mathrm{~cm}$, father $-180 \mathrm{~cm}$ ). MRI study revealed Dandy-Walker syndrome and hydrocephaly. Hypoplasia of segment A1 of the anterior cerebral artery and segment P1 of the posterior cerebral artery were revealed.

\section{Karyotypic study}

The patient has karyotype 45,X/46,X,+mar. The marker chromosome was found in $33.8 \%$ of the metaphase spreads $(N=80)$. It was small and comparable in size with chromosome 20. Although DAPI staining showed that this chromosome looks such as a small acrocentric chromosome, localization of the telomeric probe revealed no signals on it (Fig. 1). However, as shown previously, the absence of telomeres cannot indicate unambiguously that the chromosome is a ring [9]. FISH of the set of human whole chromosomes-specific probes showed the localization of chromosome $\mathrm{X}$ painting probe on the whole length of the marker chromosome (Fig. 2a). To visualize the boundaries of the derivate formation, the marker chromosome was microdissected, and the derived probe was localized on chromosomes of the patient and a healthy control. In total, we obtained 4 libraries. In the patient cells each probe painted the marker chromosome and the centromeric region of chromosome X (Fig. 2b). In the healthy control, probes painted the centromeric region of chromosome $\mathrm{X}$ only (Fig. 2c, d). This localization showed the marker chromosome was derived from region Xp11.23-q13.2. (Fig. 2c-box).

\section{PCR screening for XIST gene}

To test whether XIST gene presents in marker chromosome or not we made PCR screening of two separate copies of microdissection-derived probes of the marker chromosome (mar1 and mar2) and painting probes of chromosomes X and 3 as controls (see Materials and Methods). Both analyzed marker chromosomes do not contain XIST sequences whereas $\mathrm{X}$ centromeric sequences are presented in marker chromosomes painting probes (Fig. 3).

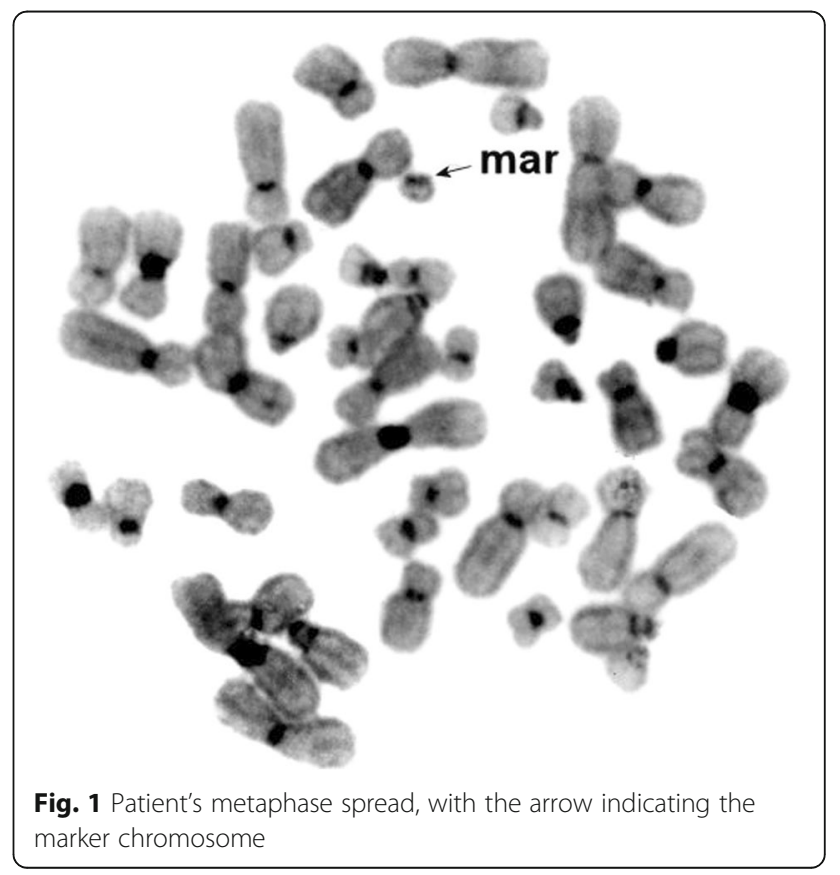



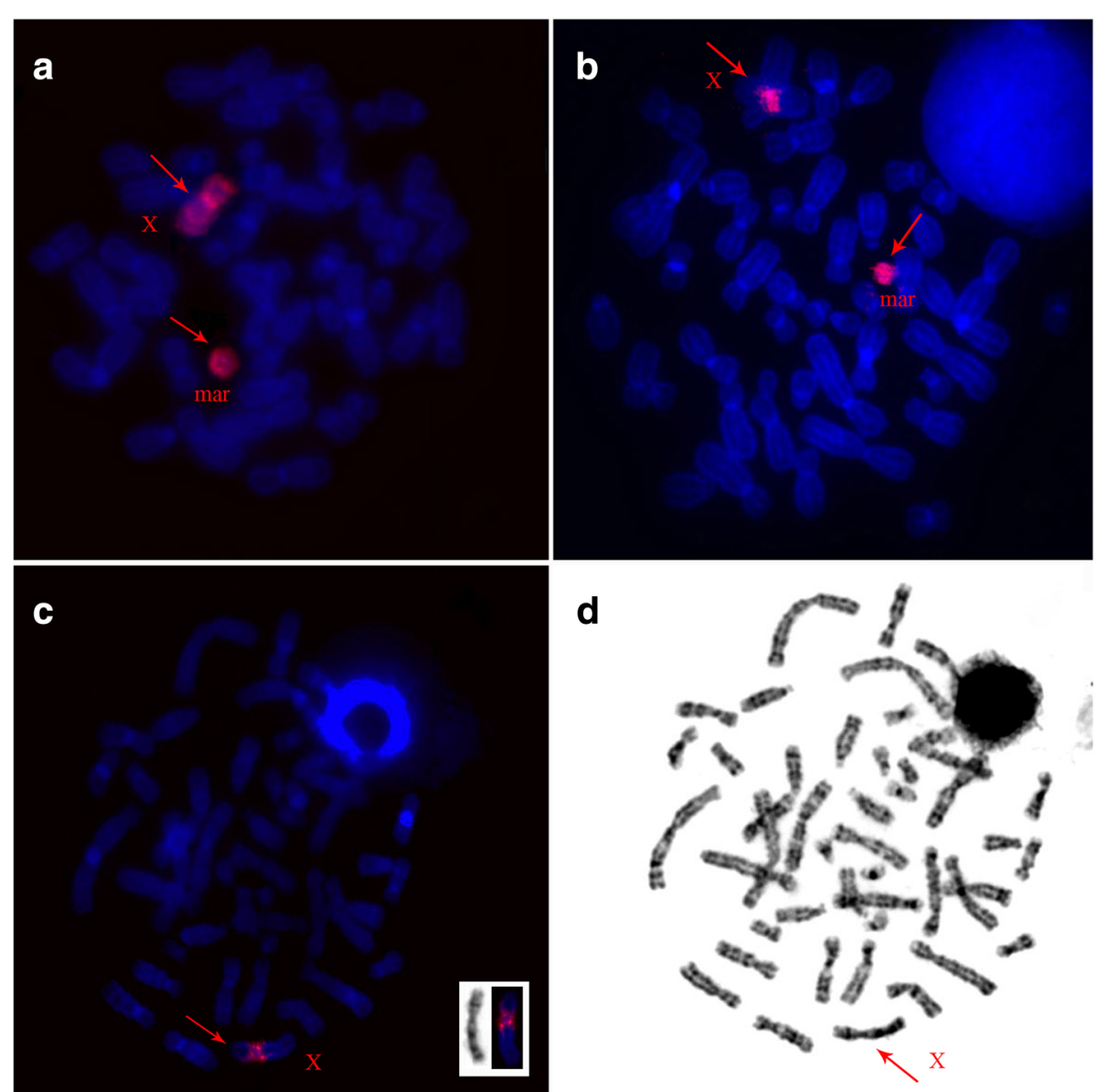

d

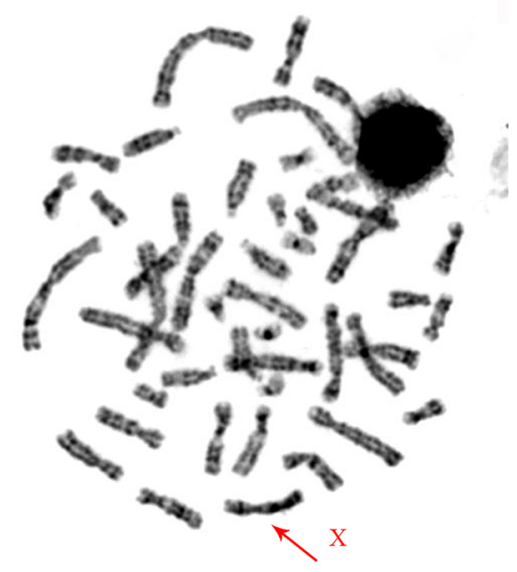

Fig. 2 Examples of fluorescent in situ hybridization: a Localization of human chromosome X painting probe (red) on chromosomes of the patient. $\mathbf{b}$ Localization of microdissected probe of marker chromosome on chromosomes of the patient. $\mathbf{c}$ Localization of microdissected probe of marker chromosome on chromosomes of the healthy control after G-banding (d). Box on (c) is localization of signal on G-banded chromosome $X$. Arrows indicate signal localization

\section{Discussion}

In 2014, Mazzaschi R. et al. described a female patient with $\mathrm{SSMC}^{\mathrm{T}}$ derived from the centromere region of the chromosome X. In that case, the proband had poor growth, global developmental delay, type I diabetes, clonic seizures, cardiomyopathy, hepatic adenomas, and skeletal dysplasia. Moreover, she had delayed secondary sexual characteristic development and ovarian failure and growth hormone deficiency [5]. The clinical symptoms of the patient in our research differed from those described above. The manifestations of Turner's syndrome in our case were milder; the patient had not expressed cognitive failure, clonic seizures or damage to other organs. However, she had Dandy-Walker syndrome. Dandy-Walker syndrome is defined by hypoplasia of the cerebellar vermis and dilation of the fourth ventricle [10]. Patients with this syndrome often have motor deficits such as delayed motor development, hypotonia and ataxia; further, approximately half have mental retardation and some have hydrocephalus [11]. Based on the results of an MRI study of the brain, hydrocephalus was diagnosed in this case as well.

A female patient with symptoms similar to the case was described earlier. The ten-year-old girl had typical manifestations of Turner's syndrome - short stature, webbing of neck, cubitus valgus, shield chest, congenital dislocation of hip, renal anomalies, clinodactyly, unilateral simian crease on right palm, acyanotic congenital heart disease and small patent ductus arteriosus. However, intellectual insufficiency did not occur in that patient, and further analysis revealed that the marker chromosome in her karyotype was derived from chromosome 14 [2].

Combining the conventional karyotyping and FISH data allowed us to characterize the genetic content of the marker chromosome. The derivate corresponds to Xp11.23-q13.2 region, which contains the gene XIST. This gene is responsible for the $\mathrm{X}$ chromosome 


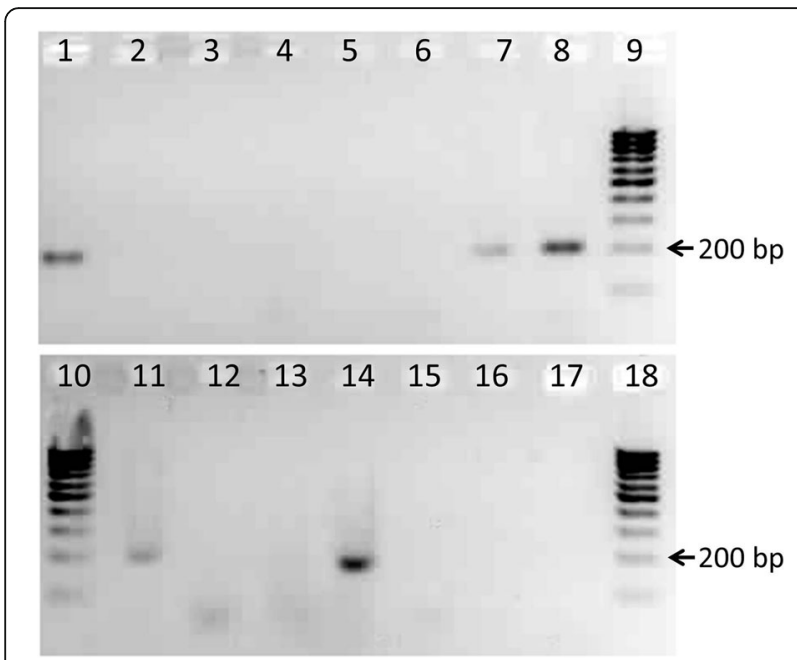

Fig. 3 Results of PCR screening of marker chromosomes for XIST gene presence. Primers: CH3R/F on lines 1-5; CHXR/F on lines 6-8, 11, 12; XISTF/R on lines 13-17. DNA templates: chromosome 3 painting probe on lines 1, 6, 13; chromosome $X$ painting probe on lines 2, 7, 14; microdissection-derived marker chromosome (mar1) on lines 3, 8, 15; microdissection-derived marker chromosome (mar2) on lines 4, 11, 16; negative controls on lines 5, 12, 17; 100 bp ladder on lines 9, 10, 18

inactivation, which is necessary to realize the epigenetic mechanism of dosage compensation. The structurally abnormal chromosome X without XIST could not be inactivated, which led to an increase in the dose of genes and, consequently, to the appearance of features of Turner's syndrome. This, in turn, led to a more severe phenotype of Turner's syndrome [5]. In the case report we found no XIST gene in X derived marker chromosome that means other reason of mild symptoms.

\section{Conclusion}

In this clinical case we described the patient with a mild phenotype of Turner's syndrome. The proband does not have intellectual disability or severe diseases of internal organs in absence of XIST gene in X-derived marker chromosome.

\section{Acknowledgements}

We thank Professor Malcolm A. Ferguson-Smith at the University of Cambridge for provided human chromosomes painting probes.

\section{Funding}

The study is supported by Russian Science Foundation Grant 15-15-10001.

\section{Ethics approval and consent to participate}

The procedure of involving the patient in the study was designed strictly in accordance with international standards, which include the awareness of the subject, his or her consent to participate in the study in its entirety, and guarantees of confidentiality. All of the studies conformed to ethical standards developed in accordance with the Helsinki Declaration of the World Medical Association as amended in 2000. In addition, the studies were supervised by the Institutional Review Board.

\section{Consent for publication}

Not applicable.

\section{Competing interests}

The authors declare that they have no competing interests.

\section{Publisher's Note}

Springer Nature remains neutral with regard to jurisdictional claims in published maps and institutional affiliations.

\section{Author details}

${ }^{1}$ Institute of Molecular and Cellular Biology SB RAS, Lavrentieva ave. 8/2, Novosibirsk 630090, Russia. ${ }^{2}$ Novosibirsk State University, Novosibirsk 630090, Russia. ${ }^{3}$ Novosibirsk State Medical University, Novosibirsk 630091, Russia. ${ }^{4}$ Novosibirsk City Clinical Hospital No.1, Novosibirsk 630047, Russia.

Received: 8 August 2017 Accepted: 3 November 2017

Published online: 17 November 2017

\section{References}

1. Liehr T, Mrasek K, Hinreiner S, Reich D, Ewers E, Bartels I, et al. Small supernumerary marker chromosomes (SSMC) in patients with a 45, X/46, X, +mar karyotype-17 new cases and a review of the literature. Sex Dev. 2007;1:353-62.

2. Sheth F, Ewers E, Kosyakova N, Weise A, Sheth J, Desai M, et al. A small supernumerary marker chromosome present in a turner syndrome patient not derived from X- or Y-chromosome: a case report. Mol Cytogenet. 2009;2:22.

3. Wang $H$, Wang $T$, Yang $N$, He $Y$, Chen $L$, Hong $L$, et al. The clinical analysis of small supernumerary marker chromosomes in 17 children with mos 45,X/46,X,+mar karyotype. Oncol Lett. 2017;13:4385-9.

4. Zhong Q, Layman LC. Genetic considerations in the patient with turner syndrome-45,X with or without mosaicism. Fertil Steril. 2012;98:775-9.

5. Mazzaschi RLP, Taylor J, Robertson SP, Love DR, George AMA. Turner syndrome patient carrying a mosaic distal $\mathrm{X}$ chromosome marker. Case Reports in Genetics. 2014:2014:597314.

6. Yudkin D, Hayward BE, Aladjem MI, Kumari D, Usdin K. Chromosome fragility and the abnormal replication of the FMR1 locus in fragile $X$ syndrome. Hum Mol Genet. 2014;23:2940-52.

7. Seabright MA. Rapid banding technique for human chromosomes. Lancet. 1971;2:971-2.

8. Liehr T. Fluorescence in situ hybridization (FISH) - application quide. 2nd ed. Berlin, Heidelberg: Springer-Verlag; 2017.

9. Guilherme RS, Klein E, Venner C, Hamid AB, Bhatt S, Melaragno Ml, et al. Human ring chromosomes and small supernumerary marker chromosomes - do they have telomeres? Chromosom Res. 2012;20:825-35.

10. Love K, Huddleston L, Olney P, Wrubel D, Visootsak J. Developmental outcomes of down syndrome and Dandy-Walker malformation. J Pediatr Neurol. 2011:9:405-8.

11. Ecker J, Shipp T, Bromley B, Benacerraf B. The sonographic diagnosis of Dandy-Walker and Dandy-Walker variant: associated findings and outcomes. Prenat Diagn. 2000;20:328-32.
Availability of data and materials

Data sharing not applicable to this article as no datasets were generated or analyzed during the current study.

\section{Authors' contributions}

AST, DVY - study concept. AST, NAL - cell growing, metaphase preparation, GTG banding, FISH experiments. SAR - microdissection. YVM, ARS - all clinical procedures and study. DVY - manuscript preparation. All authors read and approved the final manuscript. 